\title{
Der Erweiterungsbau öffnet seine Türen
}

\section{Rückblick aus Sicht der Abteilung Leserservice}

\section{Countdown der letzten Vorbereitungen}

Die letzten Tage vor der Inbetriebnahme des Erweiterungsbaus am 5. Oktober 2020 waren geprägt von vielen kleineren und größeren Arbeiten, die noch notwendig wurden, um die Bibliothek betriebsfähig zu öffnen. Insbesondere der Umzug der Benutzungsbereiche mit der kompletten Ausstattung sowie der Mitarbeiterinnen und Mitarbeiter fand während der Schließung an den drei Werktagen vor dem Wochenende statt.

Bereits in den Wochen zuvor sind in einem großen Kraftakt unter Beteiligung nahezu der gesamten Belegschaft die ca. 158.000 Bände der Freihandausleihe in der neu geordneten RVK-Aufstellung an ihrem neuen Standort eingestellt worden. Mit dem Einzug der ersten Bände in den Erweiterungsbau wurde ein Buchordnungsdienst vom Personal des Magazins gebildet, der seitdem für die Rückstellungen der Freihandausleihe und das Einstellen des Neuzugangs sowie Ordnungsaufgaben auf den vier Etagen zuständig ist. Vom Führungsteam wurden alle Mitarbeiter nach einem gestuften Schneeballkonzept durch das neue Gebäude geführt und waren somit bereits mit Erstinformationen auf die neuen Angebote und geplanten Abläufe im Service vorbereitet, so dass darauf aufbauend die Einarbeitungen für die Vertretungen in den Spätdiensten an den Servicetheken des Empfangs und der Information erfolgen konnten.

Nach diesem Marathonlauf hieß es nun, noch einen kurzen, aber kräftigen Sprint einzulegen, um die letzten Vorbereitungen zu treffen. Also packten viele emsige Hände an vielen Stellen an. Bis zur letzten Minute der Schließtage wurde noch etwas auf ungezählten Bücherwagen bewegt, eingeräumt, montiert, verkabelt, konfiguriert, getestet und geputzt, um alles für den großen Tag der Eröffnung funktionsfähig fertigzustellen und in bestem Eindruck präsentieren zu können. Angesichts der vielfältigen Aufgaben waren Tatkraft, Sorgfalt und Kompetenzen in allen Bereichen der Benutzung gefragt.
Zunächst hieß es, nach einem mit dem neu erstellten Regalplanungsmodul des 3D-Rauminformationssystems mapongo im Detail bis auf die Abbildung der Signaturbereiche ausgearbeiteten Aufstellungskonzept die ca. 70.000 Bände Präsenzbestand des ehemaligen Hauptlesesaals, die Musik- und Kunstbände sowie den Restbestand der HB Information mit Unterstützung einer Logistikfirma vom alten Gebäude an die exakten Regalstandorte in den vier Etagen zu transportieren. Am Regal wurde vom RVK-Team jeder Band in den bereits dort eingeräumten ausleihbaren Freihandbestand sorgsam einsortiert, bis zum Abschluss ein wohl geordneter Gesamtbestand das neue Erscheinungsbild der erstmals nun integrierten Freihandbestände repräsentiert. Von der Zeitschriftenstelle wurden ergänzend die Regalkästen beschriftet und der laufende Zeitschriftenjahrgang der Auslage nach der RVK-Systematik am Anfang der Fachgruppen eingeräumt.

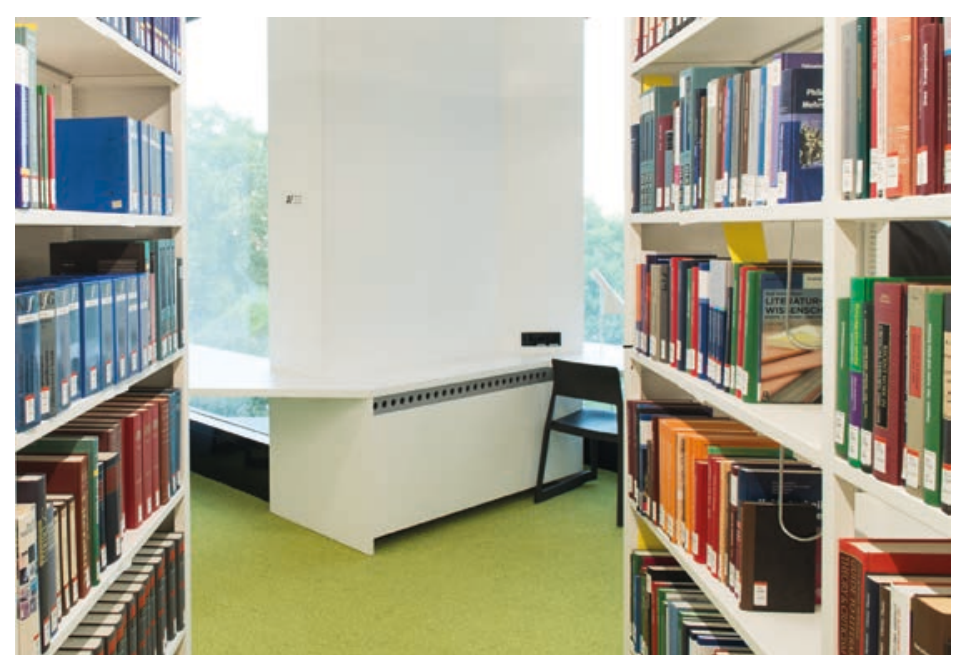

Abb. 1: Regale mit Freihandbeständen

Auch von der Ausleihe setzte sich eine Bücherwagenkolonne in Richtung des neuen Selbstabholbereiches in Bewegung. Hier wurden die abholbereiten Medien mit den Fernleihen für die Nutzer eingeräumt, nachdem die Regalbeschriftungen zuvor in dem Design des Erweiterungsbaus angebracht waren. Die Mitarbeiterinnen der Ausleihe arbeiten seitdem räumlich getrennt in zwei Arbeitsgruppen, insbesondere um die erforderlichen Abstände der 
Corona-Schutzmaßnahmen einzuhalten. Somit fanden auch alle Unterlagen und Arbeitsmaterialien ihren neuen Bestimmungsort, auch bei der Einrichtung der Empfangstheke. Shop-Artikel und Ausstellungskataloge zum Verkauf wurden besorgt und an der Empfangstheke mit weiteren Materialien zum Verkauf eingeräumt. Die erste Mitarbeiterschulung zum Betrieb der Buchförderanlage sollte eine Vorbereitung für deren Inbetriebnahme sein.
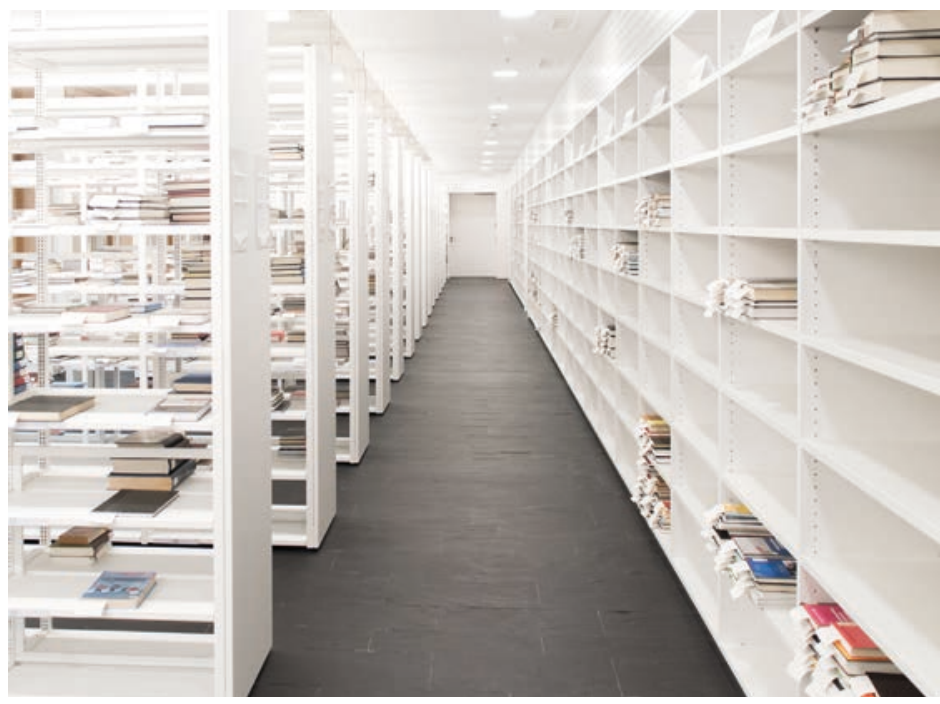

Abb. 2: Selbstabholbereich mit bereitgestellten Medien

Im Hintergrund wurden in Zusammenarbeit der Ausleihe mit der IT-Abteilung sowie der Firma aStec notwendige Anpassungen umgesetzt, die bereits für die Umsetzungsphase vorbereitet waren. Für diese Arbeiten war ein Bestellstopp ab dem Nachmittag vor Beginn des Umzugs erforderlich. An den Schließtagen wurde die Exemplar-Bestellbarkeit für die Freihandausleihe geändert, so dass diese nunmehr nicht mehr elektronisch bestellbar ist, denn die Bände sollen zukünftig vom Nutzer selbst aus den Regalen entnommen werden. Damit verbunden wurde auch die Standortangabe für jedes Medium an die vier Etagen angepasst. In einem täglichen Batch werden seitdem die Standortangaben von Neuzugängen und Umstellungen automatisch berechnet. Das erleichtert den Mitarbeitern der Medienbearbeitung die Erfassung, denn sie müssen als Standort nur "Freihand", nicht aber die genaue Etage eingeben. Ergänzend wurden von der Ausleihe die neuen Konditionen im Ausleihsystem angepasst, die Ausgabeorte für den Erweiterungsbau umbenannt, E-Mail-Vorlagen abgeändert, Briefpost ausgedruckt sowie alle aDIS-Anwendungen getestet.
Die IT-Abteilung langte kräftig zu: Mitarbeitercomputer für die Thekenbereiche und Büros mit ihrer Hardware-Ausstattung wie Zetteldrucker, RFIDAntennen, Barcodeleser und Zweitmonitore wurden umgezogen und montiert. Die Telefonanlage musste neu konfiguriert und auf den Neubau umgeschaltet werden, und die Telefone waren anzuschließen. Für den Nutzerbereich wurden Computer sowie schwere Geräte wie Aufsichtsscanner und Kassenautomaten bewegt. Selbstverbuchungsterminals, Multifunktionsgeräte und Readerprinter mussten an ihrem neuen Platz installiert werden. Die Gates zur Buchsicherung und die Selbstverbuchungsgeräte wurden neu konfiguriert, außerdem eine Shopfunktion für den Kassenautomaten eingerichtet. Anschließend wurde von der Ausleihe und der IT umfangreich getestet.

Für das ehemalige Lesesaalteam galt es zeitgleich, die nicht ausleihbaren Medien und Fernleihen der Rücklage zu transportierten und wieder einzuräumen. Diese finden nun ihren Platz in einer Kompaktregalanlage. Auch die Mikroformen wurden wieder fein säuberlich geordnet in Schubladen einsortiert, die Materialien der Theke mit den Schalterwerken sowie Beilagen und CD-ROMs der Präsenzbestände und die Semesterapparate eingeräumt. Alles fand schließlich seinen Platz und steht ordentlich beschriftet zur Nutzung zur Verfügung.

Die Integration des Personals des Lesesaals und der Information in einem Team ist nicht nur im Thekenbereich der Information in der ersten Etage sichtbar. Die Vollzeitkräfte arbeiten in einem gemeinsamen Büro zusammen. Alle Arbeitsmaterialien und Aktenordner wurden hier oder direkt an der Informationstheke untergebracht. Bereits im Vorfeld wurde vieles vorbereitet, was entsprechend seinen Abschluss fand. Das Arbeitsplatzreservierungssystem WLB-Riserva konnte im Echtbetrieb an den Terminals getestet werden. Erforderliche CoronaBeschilderungen, Aushänge und Wegeführungen wurden angebracht, die temporär als Ergänzung des fest montierten Leitsystems vorgesehen sind. Die Beschriftungsfirma, die alle Ausschilderungen sowie die Tischnummern anbrachte, wurde betreut.

Alle wichtigen Informationen in der Außenkommunikation wurden von der Website-Redaktion auf die Homepage gestellt und vom Social-Media-Team im 
Blog, Facebook, Instagram und Twitter verbreitet. EMail-Vorlagen wurden aktualisiert sowie die Anrufbeantworter jeweils auf den neuesten Stand besprochen. Für die interne Benutzerinformation wurden Ausdrucke für die RVK-Aufstellung nach Etagen vorbereitet und es fand im Team die letzte intensive Einarbeitungsphase zur Benutzerberatung statt.

Am Ende dieser drei arbeitsintensiven Tage hieß es in allen Arbeitsbereichen am Freitagabend: Es ist geschafft! Die große Erleichterung war allen ins Gesicht geschrieben. So fielen auch die wenigen Lücken - wie die noch nicht funktionsfähige Buchförderanlage - nicht besonders auf. Alle waren stolz auf den Erweiterungsbau und die damit verbundenen Serviceangebote, die mit viel Aufmerksamkeit sowie Ideenreichtum bedacht und unter großem Einsatz neben der Aufrechterhaltung des täglichen Betriebs vorbereitet wurden. Ein gewisser Wermutstropfen war jedoch für alle damit verbunden, dass die Corona-Infektionslage es erforderlich machte, nicht alle Einzel- und gar keine Gruppenarbeitsplätze anbieten zu können. Die Wegeführung in der Bibliothek musste so geändert werden, dass der Treppenaufgang nicht über die Haupttreppe und damit in der Nähe der Information stattfinden konnte. Auch wird der Zugang im Eingangsbereich weiterhin über einen Wachdienst kontrolliert.

Trotz der enormen Kraftanstrengungen startete ein stark motiviertes Team der Abteilung Leserservice in das Wochenende, um noch etwas Kraft für die folgende Woche der Inbetriebnahme zu schöpfen.

\section{Inbetriebnahme des Erweiterungsbaus}

Die Spannung am Eröffnungstag des 5. Oktobers 2020 war schließlich hoch und alle hatten eine erwartungsvolle Vorfreude, die neuen Servicebereiche und vier Bücheretagen für die vielen zu erwartenden Besucher des schönen Erweiterungsbaus in Betrieb zu nehmen!

Gleich um 8 Uhr mit der Öffnung der Eingangstüren strömten die ersten Besucher in die Bibliothek und seitdem hat dieser Zustrom nicht nachgelassen. Die große Zahl der Nutzer in Zeiten von CoronaEinschränkungen beweist, dass der Neubau schnell seinen Platz in der Stuttgarter Innenstadt gefunden hat und sich bereits einer großen Beliebtheit erfreut.
Der Erweiterungsbau überraschte viele ob seiner Größe und Weitläufigkeit in den Etagen mit einem einmaligen Ausblick auf das Neue Schloss und den

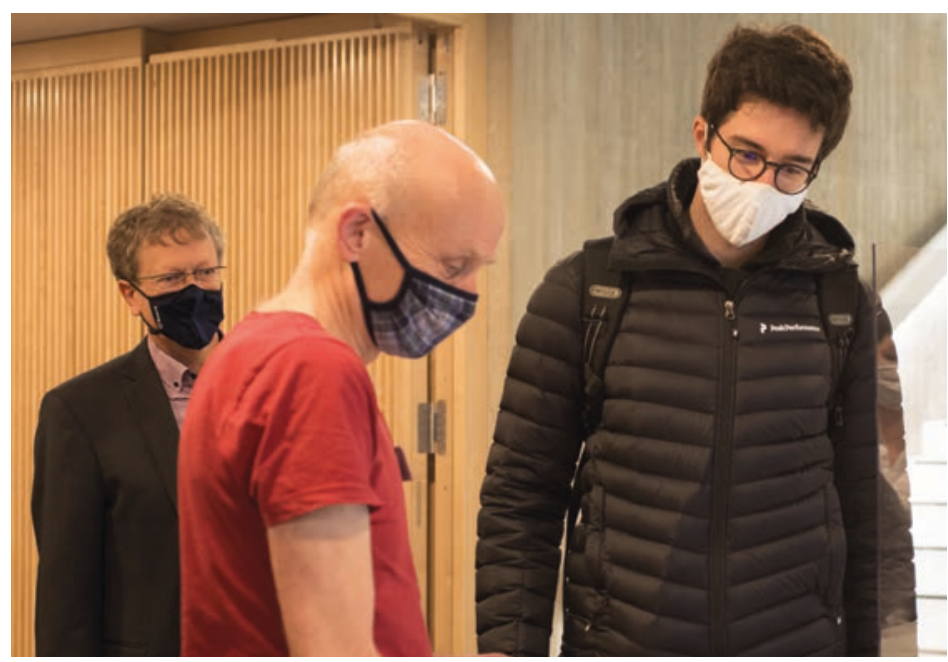

Abb. 3: Begrüßung des ersten Nutzers (r.)

Landtag. Die vielen Nutzerplätze und Regalreihen voller Bücher erfreuen nochmals mehr alle, die bereits zuvor gerne die WLB zur Ausleihe und als Lernort genutzt haben. Das Einrichtungskonzept wurde vielfach gelobt und die Aufenthaltsqualität mit den umfangreichen Arbeitsplätzen von der ersten Stunde an besonders geschätzt. Auch wenn die Wegeführung zum gebuchten Arbeitsplatz aufgrund der coronabedingten Änderungen zunächst nicht allen einfach fiel, haben die meisten bald ihren Lieblingsarbeitsbereich in der Nähe ihrer fachlichen Bestände auf den Etagen gefunden.

\section{Der Erweiterungsbau im Feedback des Publikums}

Das große Nutzerinteresse an der Bibliothek spiegelt sich auch in den zahlreichen Rückmeldungen

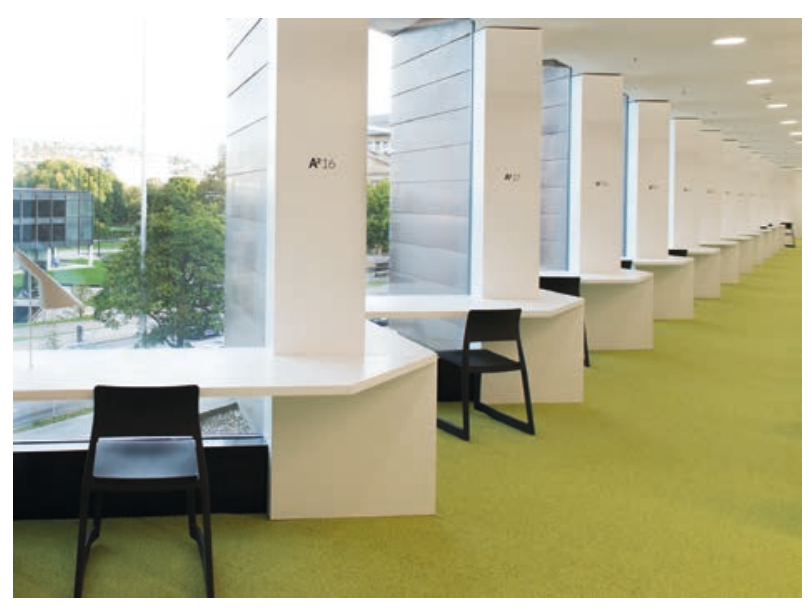

Abb. 4: Arbeitsplätze mit Blick auf den Landtag 
wider, die anhand einer ausgelegten Umfrage, per E-Mail und auch persönlich direkt bei den Mitarbeitern eingingen. Alle Verbesserungsanregungen sind bereits geprüft (wie die Außenbeschilderung zum neuen Haupteingang, Anpassung der Raumtemperatur an Jahreszeiten...) und wenn noch nicht umsetzbar, dann doch schon in die offene Liste aller Nacharbeiten (wie Verbesserung des WLANs, Cafeteria-Angebot als Pausenbereich) aufgenommen worden.

Ein treffendes Zitat zum Zweck einer Bibliothek wird Henry Ward Beecher (1813-1887) zugeschrieben: „Eine Bibliothek ist kein Luxus, sondern eine Notwendigkeit des Lebens." Auch wenn der Erweiterungsbau zunächst den notwendigen und dringenden Bedarf der WLB-Nutzer erfüllen soll, so soll auch seine architektonische Gestaltung und Inneneinrichtung mit Ästhetik verbunden sein, damit das Lernen und Verweilen in den Lesebereichen zu einem persönlichen Gefühl von Luxus werden darf. Einige Stimmen unseres Publikums sollen daher zum Abschluss diesen Eindruck ihrer Wahrnehmung und Wertschätzung vermitteln:

\section{„Wenn man einmal alles Organisatorische geklärt} hat, ist das System super strukturiert, verständlich und sinnvoll. Bei mir hat gleich beim ersten Besuch alles bestens funktioniert. Die Empfangsdamen waren sehr hilfsbereit und ich konnte erfolgreich an meinem gebuchten Arbeitsplatz arbeiten. "

„Mein erster Eindruck war Staunen und Begeisterung. Die Arbeitsplätze mit Blick auf den Landtag sind wunderschön geworden, sehr groß und mit Stromanschluss. Auch die Organisation der Freihandbibliothek gefällt mir sehr gut, vor allem die verschiebbaren Regale ... Daumen hoch!"

„Die Landesbibliothek ist ein toller Platz zum Arbeiten und Lernen. Alles ist modern \& neu. Das Personal ist sehr freundlich ..."

"Architektonisch ist die neue Landesbibliothek überraschenderweise sehr beeindruckend. Die Farben (weiß, grau, grüner Fußboden) stellen eine ruhige, sachliche und gedämpfte Stimmung her. Ich bin sehr glücklich darüber, dass die Bücher als Freihandbestand zugänglich sind und über die Offenheit, die durch die Fensterfassaden nach außen (sowie nach innen) die Räumlichkeiten lichtdurchfluten und freundlich gestalten..."

"Liebe Bibliothek, ich bin wirklich sehr begeistert. Die große Anzahl an Arbeitsplätzen mit einer absolut angenehmen Atmosphäre. Ruhe, Abstand und Ausblick sind fantastisch. Vielen Dank!"

„Gut Ding will Weile haben! Endlich steht der Neubau der WLB und ist besuchsoffen. Ich bin beeindruckt von der Benutzerfreundlichkeit der neuen Räume!"

"Ich bin total begeistert. Die neue Bibliothek ist einfach wunderschön! Das schlichte, moderne Design und der tolle Ausblick sorgen für eine gute Arbeitsatmosphäre. Die neuen Stühle sind auch einfach super ..."

"Beeindruckende Arbeit von Arno Lederer! Wahrscheinlich der zurzeit schönste Arbeitsplatz zum wissenschaftlichen Arbeiten in Stuttgart. Die Strukturierung der Sitzplätze ist perfekt (in Corona-Zeiten). "

„Ein tolles Gebäude und endlich die Möglichkeit, an genügend modernen Arbeitsplätzen zu lernen, zu recherchieren und zu arbeiten. Zusätzlich ein erstklassiges Hygienekonzept ..."

"Mit dem Hygienekonzept und Service in der WLB bin ich rundum zufrieden ... Vielen Dank!"

Christine Herrmann

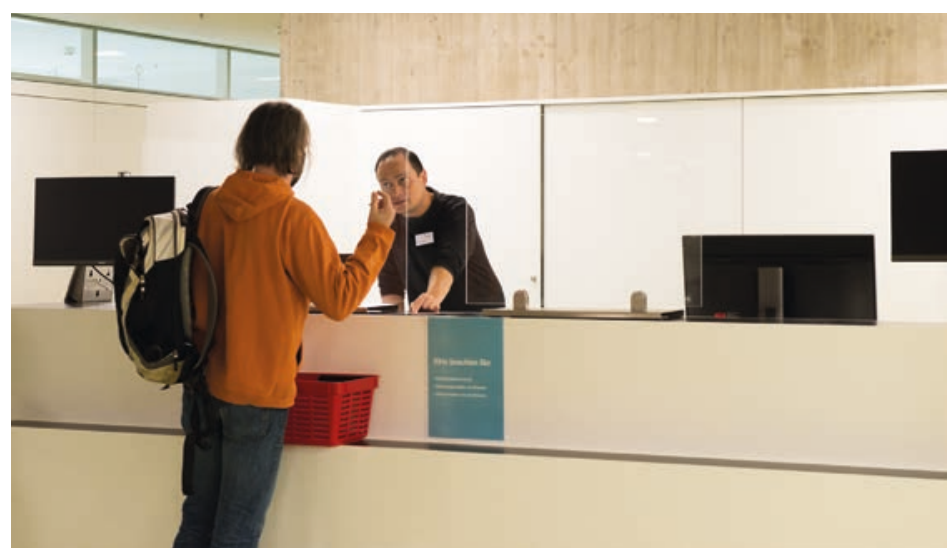

Abb. 5: Informationstheke in der ersten Etage 